\title{
Auditory P300 in Noise in Younger and Older Adults
}

DOI: $10.3766 /$ jaaa. 17077

Jennifer McCullagh*

Jennifer B. Shinn $\dagger$

\begin{abstract}
Background: Older adults often report difficulty hearing in background noise which is not completely attributable to peripheral hearing loss. Although age-related declines in cognition and hearing in background noise occur, the underlying age-related changes in processing of auditory stimuli in background noise has yet to be fully understood. The auditory $\mathrm{P} 300$ has the potential to elucidate the effects of age on auditory and cognitive processing of stimuli in background noise, but additional research is warranted.
\end{abstract}

Purpose: The purpose of this study was to investigate age-related differences in cognitive processing of auditory stimuli by evoking the auditory P300 at multiple signal-to-noise ratios (SNRs).

Research Design: A two-group, repeated measures study design was used.

Study Sample: A convenience sample of 35 participants, 15 older adults (mean age of $66.4 \mathrm{yr}$ ) and 20 younger adults (mean age of $21.1 \mathrm{yr}$ ), participated in the study. All participants had negative otologic and neurological histories.

Data Collection and Analysis: The auditory P300 was evoked using an oddball paradigm with 500 (frequent) and $1000 \mathrm{~Hz}$ (target) tonal stimuli in quiet and in the presence of background noise at $+20,+10$, and 0 SNRs. P300 amplitudes and latencies were measured in each condition for every participant. Repeated measures analyses of variance were conducted for the amplitude and latency measures of the P300 for each group.

Results: Results from this study demonstrated P300 latencies were significantly longer in older adults in noise at the most challenging condition ( 0 SNR) compared with the quiet condition and between the +10 SNR and 0 SNR conditions. Although older adults had significantly longer P300 latencies compared with younger adults, no significant group by listening condition interaction existed. No significant P300 amplitude differences were found for group, noise, or group $\times$ listening condition interactions.

Conclusions: Results provide evidence that auditory cortical processing, regardless of age, is poorer at more difficult SNRs. However, results also demonstrate that older adults perform significantly poorer than younger adults. This supports the notion that some degree of age-related decline in synchronous firing and rate of transmission of the auditory cortical neurons contributing to the auditory P300 exists. Studies are needed to further understand the impact of noise on auditory cortical processing across populations.

Key Words: aging, cognition, hearing in noise, P300

Abbreviations: ANOVA = analyses of variance; OA = older adult; SNR = signal-to-noise ratio; $\mathrm{YA}=$ younger adult

\section{INTRODUCTION}

$\mathrm{O}$ lder adults (OAs) often report difficulty hearing in background noise which is not completely attributable to peripheral hearing loss (Dubno et al,
1984; Gordon-Salant, 1987; Stuart and Phillips, 1996; Gordon-Salant and Fitzgibbons, 1997; Snell et al, 2002; Pichora-Fuller, 2003). Researchers have investigated other age-related changes that might impact the ability to hear in background noise. Investigators have

*Southern Connecticut State University, New Haven, CT; †University of Kentucky Medical Center, Lexington, KY

Corresponding author: Jennifer McCullagh, Department of Communication Disorders, Southern Connecticut State University, New Haven, CT 06515; Email: mccullaghj1@southernct.edu

Portions of this article were presented at the American Speech, Language, Hearing Association Convention 2010 in Philadelphia, PA, the Global Perspective on CAPD conference in 2012 in Boston, MA, and AudiologyNOW! 2012, the annual convention of the American Academy of Audiology, in Boston, MA. 
demonstrated that hearing in background noise is a complex process that necessitates the use of multiple systems, including cognition (Pichora-Fuller et al, 1995; Tun et al, 2002; Anderson et al, 2013; Helfer and Freyman, 2014). Although age-related declines in cognition and hearing in background noise occur, the underlying age-related changes in the processing of auditory stimuli in background noise are yet to be fully understood.

Auditory cortical potentials, specifically N1 and P2, have been used to objectively document changes in auditory cortical processing in background noise in younger adults (YAs) and OAs. The N1 and P2 potentials have long been reported to be exogenous potentials with neural generators in the auditory cortices (Vaughan and Ritter, 1970; Elberling et al, 1982; Scherg and Von Cramon, 1986; Scherg et al, 1989). Researchers have reported significantly longer N1 and P2 latencies when comparing quiet with noise conditions in YAs (Billings et al, 2009; McCullagh et al, 2012) and OAs (McCullagh and Shinn, 2013; Billings et al, 2015) as well as significantly longer P2 latencies for OAs compared with YAs (McCullagh and Shinn, 2013). Significantly reduced amplitudes of N1 (Billings et al, 2009) and P2 (Billings et al, 2009; McCullagh et al, 2012) in noise compared with quiet conditions in YA listeners have also been demonstrated. Significantly larger N1 and P2 amplitudes were found for OAs compared with YAs in the quiet condition (McCullagh and Shinn, 2013; Billings et al, 2015). Conflicting evidence exists regarding differences in noise between YAs and OAs. Although Billings and colleagues (2015) demonstrated significant amplitude differences in noise when comparing OAs and YAs, others have found no significant differences between groups in the poorest noise condition tested [0 signal-to-noise ratio (SNR)] (Kim et al, 2012; McCullagh and Shinn, 2013). Methodological variations likely led to the differences in findings across studies; however considered together, these findings suggest that auditory cortical responses vary in noise, at least to some degree, in YAs as compared to OAs.

Converse to the auditory N1 and P2, the auditory P300 has long been known to be an endogenous potential and has the ability to establish the cognitive aspects of processing auditory stimuli (Sutton et al, 1965; Goodin et al, 1983; Squires and Hecox, 1983; Kibbe-Michal et al, 1986). It should be noted, however, evidence exists to support contributions that are exogenous in nature as well (Stapleton and Halgren, 1987; Stapleton et al, 1987; McCullagh et al, 2009). The P300 is elicited using an oddball paradigm, and the listener must make a decision regarding the stimuli to produce a response (Sutton et al, 1965; Stapleton et al, 1987), and P300 latencies are extended as the complexity of the task becomes more challenging (Polich, 1987; Gaál et al, 2007). Findings suggest that as task complexity increases, a delay in cognitive processing of the auditory stimuli occurs. These findings have implications for the processing of more complex auditory stimuli in the presence of noise.

Investigators have researched the complex task of listening in background noise using the auditory P300 in adult listeners. They have demonstrated significantly longer P300 latencies (Polich et al, 1985; Obert and Cranford, 1990; Salisbury et al, 2002; McCullagh et al, 2012) and reduced P300 amplitudes (Obert and Cranford, 1990) when background noise was present in adults. Although McCullagh et al (2012) included only YA listeners in their study, both Obert and Cranford (1990) and Salisbury et al (2002) included young and middle-aged adults in their investigations. They consistently demonstrated delays in cognitive processing of auditory stimuli in background noise in adult listeners; however, age-related changes in processing were not specifically addressed in these studies.

Age-related changes in P300 in quiet conditions have been observed (Goodin et al, 1978; Pfefferbaum et al, 1980; 1984; Picton et al, 1984; Anderer et al, 1996). Significantly longer P300 latencies and reduced P300 amplitudes in OA listeners as compared to YA listeners were reported (Goodin et al, 1978; Picton, et al, 1984; Anderer et al, 1996). Conversely, others did not find significant reductions in P3 amplitude between OAs and YAs (Pfefferbaum et al, 1980). Differences between these outcomes are likely related to age-related hearing loss, stimulus presentation levels, and recording electrode site differences between studies. These results do, however, suggest some age-related declines in synchronous firing and rate of transmission of the auditory cortical neurons contributing to the P300 potential (reviewed by Brody et al, 1975; Goodin et al, 1978).

Although age-related changes in the auditory P300 have been reported in quiet, little data in the literature exist regarding the age-related changes in the P300 in background noise. Although no significant age-related changes in P300 latency in contralateral noise were reported (Cranford and Martin, 1991; Bertoli et al, 2005), some researchers have reported significant age effects for P300 amplitude in contralateral noise (Bertoli et al, 2005), whereas others have not (Cranford and Martin, 1991). These two studies were limited by the utilization of monaurally presented stimuli in contralateral noise only. Because hearing in noise is typically a binaural process, the binaurally recorded P300 allows for a better determination of the age-related changes in cognitive processing of auditory stimuli in background noise. Furthermore, the methodologies of these studies are limited by the utilization of one SNR. Eliciting the P300 at multiple SNRs allows for the comparison of the changes that occur in cognitive processing of the auditory stimuli as the task complexity increases (i.e., decreasing SNR). Given that conflicting evidence exists related to the changes in cognitive processing of auditory stimuli and a lack 
of evidence exists related to binaurally-recorded P300 evoked responses at multiple SNRs, additional research is warranted. Therefore, the purpose of this study was to investigate age-related differences in cognitive processing of auditory stimuli by evoking the auditory P300 at multiple SNRs.

\section{METHODOLOGY}

\section{Participants}

Thirty-five adults were recruited from the University and neighboring communities to participate in this study. All participants reported negative otologic and neurological histories. Otoscopy was within normal limits bilaterally for all participants. Participants were divided into a YA group $(\mathrm{n}=20$; mean age $=21.1 \mathrm{yr}$, standard deviation $=2.7 \mathrm{yr})$ and an OA group $(\mathrm{n}=$ 15 ; mean age $66.4 \mathrm{yr}$, standard deviation $=4.6 \mathrm{yr}$ ). All OA participants were administered a backward digit span task to assess working memory and had to perform within normal limits (Gregoire and Van der Linden, 1997) to be included in the study. Data from the normalhearing YA group were previously reported in McCullagh et al (2012). The Southern Connecticut State University Institutional Review Board approved this study, and all participants signed an informed consent form before testing.

Hearing sensitivity for all groups was established using the modified Hughson-Westlake procedure (Carhart and Jerger, 1959). All participants presented with no airbone gaps greater than $10 \mathrm{~dB} \mathrm{HL}$ and no interaural asymmetries greater than $15 \mathrm{~dB}$ HL. All YA participants had pure tone thresholds better than $25 \mathrm{~dB}$ HL for the octave frequencies of 250 to $4000 \mathrm{~Hz}$ and for $6000 \mathrm{~Hz}$, bilaterally. All OA participants had normal hearing sensitivity for the octave frequencies of 250 to $1000 \mathrm{~Hz}$ and thresholds no poorer than $55 \mathrm{~dB}$ HL at 2000, 4000, and $6000 \mathrm{~Hz}$, bilaterally. All participants had normal hearing sensitivity at the two frequencies used in the electrophysiological procedure (500 and $1000 \mathrm{~Hz}$ ). Mean audiometric data for the YA and OA groups are presented in Table 1. Mean thresholds were within $10 \mathrm{~dB}$ HL at 500 and $1000 \mathrm{~Hz}$ between groups. Results from independent $t$ tests demonstrated no statistically significant differences between the two groups for the left ear $500 \mathrm{~Hz}$ thresholds $(t=-0.18, p=0.86)$. Results did demonstrate statistically significantly differences between the two groups for the thresholds for the following: left ear at $1000 \mathrm{~Hz}(t=-4.38, p<0.001)$; right ear at $500 \mathrm{~Hz}(t=-5.25, p<0.001)$; right ear at 1000 $\mathrm{Hz}(t=-3.73, p<0.001)$.

\section{Procedures}

All participants were seated in a double-walled sound treated booth. N1, P2, and P300 were measured using an Intelligent Hearing Systems (IHS) SmartEP system with silver chloride cup electrodes affixed to $\mathrm{Cz}$ (noninverting) and the nape of the neck (inverting). Ground and eye-blink electrodes were affixed to the high forehead and outer canthus of the eye, respectively. Impedances were below $3 \mathrm{k}$ ohms and balanced across electrode sites. Only one noninverting electrode $(\mathrm{Cz})$ was used to establish a protocol that was considered clinically feasible. Neuroelectric activity was filtered from 1 to $30 \mathrm{~Hz}$ with a $12 \mathrm{~dB} /$ octave roll-off. A standard oddball paradigm ( $80 \%$ frequent tones; $20 \%$ target tones) was used to evoke the potentials. Forty millisecond (10 msec rise/fall; 20 msec plateau) $500-\mathrm{Hz}$ stimuli (frequent) and $1000-\mathrm{Hz}$ (target) stimuli were presented binaurally at a repetition rate of 0.9 per sec. Responses were obtained binaurally for the purposes of ecological validity and to replicate "real-world" listening environment. Participants were asked to count the number of $1000-\mathrm{Hz}$ (target) tones presented for each trial. All waveforms were replicated and an average of the two waveforms (approximately 600 trials total; 300 original and 300 replicated) was used for amplitude and latency measurements. The number of rejected trials was maintained at less than $10 \%$ for inclusion of data. N1 and P2 data were collected and those results were previously reported in McCullagh and Shinn (2013).

The P300 waveforms were acquired in four listening conditions (quiet, $+20,+10$, and $0 \mathrm{SNR}$ ). Listening condition order was counter-balanced across participants. Binaural behavioral thresholds were established to the $500-$ and $1000-\mathrm{Hz}$ stimuli generated by the Intelligent Hearing Systems (IHS) SmartEP system as well as the white noise generated by the Grason Stadler Inc (GSI) 61 diagnostic audiometer to establish the different SNRs. The 500- and $1000-\mathrm{Hz}$ stimuli were routed through ER-3A

Table 1. Audiometric Data Including Mean 500- and 1000-Hz Thresholds, three-Frequency Pure Tone Averages (PTA) and High Frequency Pure Tone Averages (HFPTA) for the YA and OA Groups

\begin{tabular}{|c|c|c|c|c|c|c|c|c|}
\hline & \multicolumn{4}{|c|}{ Left Ear } & \multicolumn{4}{|c|}{ Right Ear } \\
\hline & $500-\mathrm{Hz}$ & $1000-\mathrm{Hz}$ & PTA & HFPTA & $500-\mathrm{Hz}$ & $1000-\mathrm{Hz}$ & PTA & HFPTA \\
\hline YAs & 5.67 & 4.00 & 4.56 & 4.67 & 7.33 & 4.00 & 4.33 & 4.22 \\
\hline OAs & 12.33 & 11.33 & 13.11 & 17.11 & 13.67 & 12.33 & 15.11 & 19.33 \\
\hline
\end{tabular}


insert earphones with open-fit hearing aid domes affixed on the ends to keep the ear canals free from occlusion (Weihing and Musiek, 2008). For the noise conditions, continuous white noise was presented through a speaker positioned at zero degrees azimuth 42 " from the participants' nose. The $500-$ and $1000-\mathrm{Hz}$ stimuli were presented at $50 \mathrm{~dB}$ SL re: behavioral tone burst threshold, and the SNRs in the noise conditions were achieved by altering the white noise intensity levels (McCullagh and Shinn, 2013). Before initiation of the protocol, levels were calibrated using a sound level meter to ensure accurate SNRs.

P300 amplitudes and latencies were measured for each listening condition (quiet, $+20,+10$, and $0 \mathrm{SNR}$ ). P300 was considered the most positive peak between 245 and 450 msec poststimulus onset. When a bifid or broad peak was present, the midpoint of the waveform was used as the latency measurement. P300 amplitude was measured peak-to-trough from the highest peak of P300 to the closest negative trough following P300 (Jirsa, 1992; McCullagh et al, 2012). Waveforms were analyzed by two experienced researchers with extensive experience in auditory late potentials. One of the two reviewers was blinded to the condition in which the waveforms were acquired.

\section{Statistical Design}

Repeated measures analyses of variance (ANOVA) were conducted for the amplitude and latency measures of the P300. The analyses were performed using SPSS statistical software (SPSS, Inc., Release 19.0.0). The analyses included the within-subjects factor of listening condition (quiet, $+20,+10$, and $0 \mathrm{SNR}$ ) and betweensubjects factor of group (YA and OA). GreenhouseGeisser corrected degrees of freedom were used when sphericity could not be assumed (Greenhouse and Geisser, 1959).

Post hoc paired $t$-tests were conducted to determine where significant differences within participants existed. Paired $t$-tests for all combinations of listening conditions were conducted. In addition, planned comparisons were performed for the following conditions: quiet and $+20,+20$ and $+10 \mathrm{SNR},+10$ and $0 \mathrm{SNR}$, and quiet and 0 SNR. Bonferroni adjustments were performed at the alpha level of 0.05 to adjust for the number of $t$-tests conducted for each measure.

\section{RESULTS}

\section{Descriptive Statistics}

Descriptive statistics for P300 latencies and amplitudes for each group at each SNR (quiet, $+20,+10$, and 0 SNR) are displayed in Figures 1 and 2. Mean P300 latencies increased from the quiet condition to

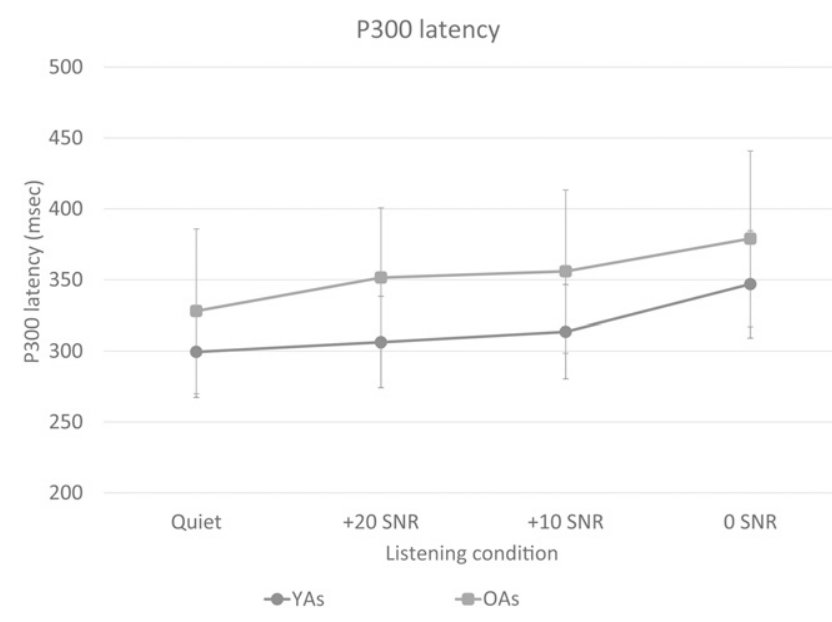

Figure 1. Mean P300 latencies for the older and younger adult groups in quiet and at $+20,+10$, and 0 SNRs.

the 0 SNR condition for each group. Mean P300 latencies were longer for the OA compared with the YA group in each listening condition. Mean P300 amplitudes decreased for the YA group, but remained relatively stable for the OA group in each listening condition. Mean P300 amplitudes were between 0.6 and $2.1 \mu \mathrm{V}$ greater for the YA group in the each of the listening conditions, with the greatest differences in amplitude occurring in the quiet condition.

\section{Latency}

Repeated measures ANOVAs were conducted to determine if significant differences occurred for P300 latencies in the different listening conditions (quiet, +20 , +10 , and 0 SNR) between groups. Results of the repeated measures ANOVAs indicated significant latency differences between listening conditions $\left[F_{(1,3)}=17.03\right.$, $p<0.001]$. Latencies were significantly longer for the

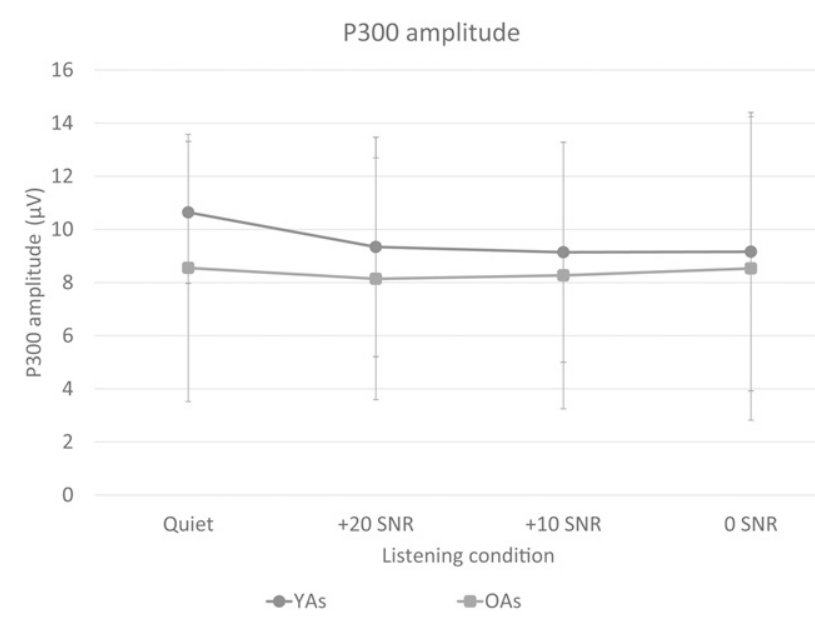

Figure 2. Mean P300 amplitudes for the older and younger adult groups in quiet and at $+20,+10$, and 0 SNRs. 
OA compared with the YA groups $\left[F_{(1,3)}=8.48, p=\right.$ $0.01]$; however, no significant group $\times$ listening condition interactions existed $\left[F_{(1,3)}=0.65, p=0.59\right]$.

Post hoc comparisons were conducted for the different noise levels to determine where significant differences existed. The result of the Bonferroni adjustment was an alpha level of 0.0125 . Significant latency differences did not exist between the quiet and +20 SNR conditions $(t=-2.20, p=0.03)$, or the +20 and +10 SNR conditions $(t=-0.86, p=0.40)$. However, significantly longer P300 latencies existed in the 0 SNR condition compared with the quiet condition $(t=-5.99, p<0.001)$ and the 0 SNR condition compared with the +10 SNR condition $(t=-4.77, p<0.001)$.

\section{Amplitude}

Repeated measures ANOVAs were conducted to determine if significant differences existed for the P300 amplitudes in the different listening conditions (quiet, +20 , + 10, and 0 SNR) between the two groups. Results of the repeated measures ANOVAs indicated no significant amplitude differences between listening conditions $\left[F_{(1,3)}=0.86, p=0.46\right]$. Amplitudes were not significantly different for the YA compared with the OA group $\left[F_{(1,3)}=0.79, p=0.38\right]$. No significant group $\times$ listening condition interactions existed $\left[F_{(1,3)}=0.50, p=0.68\right]$.

\section{DISCUSSION}

$\mathrm{T}$ he purpose of the study was to ascertain the agerelated changes in the auditory P300 in background noise. P300 responses were elicited in YAs and OAs by binaurally presenting stimuli in quiet and at three SNRs $(+20,+10$, and 0 SNR). Results from this study demonstrated P300 latencies were significantly longer in OAs in noise at the most challenging condition (0 SNR) compared with the quiet condition and between the +10 and 0 SNR conditions. Although OAs had significantly longer P300 latencies compared with YAs, no significant group by listening condition interaction existed. No significant P300 amplitude differences were found for group, noise, or group $\times$ listening condition interactions.

Although the effects of peripheral hearing cannot be completely ruled out, attempts were made to minimize the impact of this potential confounding variable. All participants in the YA and OA groups had normal peripheral hearing sensitivity for the two test stimuli (500 and $1000 \mathrm{~Hz}$ ). The majority $(66 \%)$ of the OA participants had bilaterally normal peripheral hearing sensitivity from the octave frequencies of 250 to $4000 \mathrm{~Hz}$ and the interoctave, $6000 \mathrm{~Hz}$. In addition, the test stimuli were presented at $50 \mathrm{~dB}$ SL re: 500 and $1000 \mathrm{~Hz}$ thresholds to further minimize the impact of audibility on the study outcomes.
P300 latencies were significantly longer in noise than in quieter conditions. Longer P300 latencies were found between the poorer, and likely more auditory demanding, SNRs (+10 and 0 SNR; quiet and 0 SNR). These findings are similar to previous studies which demonstrated prolonged P300 latencies in background noise (Polich et al, 1985; Obert and Cranford, 1990; Salisbury et al, 2002; McCullagh et al, 2012). Investigators have also previously indicated that P300 latencies increase with greater task complexity suggesting delayed cognitive processing with more challenging conditions (Polich, 1987; Gaál et al, 2007). Because P300 latencies do not significantly increase until more noise is present (0 SNR), it suggests that cognitive processing of the auditory stimuli remains relatively stable at easier SNRs, but becomes significantly delayed (poorer) at the most difficult listening condition (0 SNR).

P300 latencies were significantly longer for OAs than YAs, but a significant group by listening condition interaction did not exist. Significantly longer P300 latencies in OAs compared with YAs have been previously reported (Cranford and Martin, 1991; Bertoli et al, 2005; Gaál et al, 2007; Kropotov et al, 2016) and indicated an agerelated slowing of processing and/or increased inhibition which contributes to the increases in P300 latency (Papanicolaou et al, 1984; Peters, 2002; Gaál et al, 2007). Because P300 latency has been attributed to stimulus processing rather than response processing (reviewed by Rossini et al, 2007), P300 latency increases in older compared with YAs suggest that OAs take longer to classify stimuli than YAs. The current study demonstrated the lack of a significant interaction between age and listening condition on the P300 latency, suggesting that as the SNR decreases, processing time increases across ages in general. Other researchers have also failed to report significant interactions between age and P300 latencies in background noise (Cranford and Martin, 1991; Bertoli et al, 2005). Thus, support exists for a reduction in stimulus processing speed related to age, but cognitive processing in background noise is not more delayed in OAs than in YAs.

One unexpected finding was that no statistically significant P300 amplitude differences for group, noise, or group $\times$ listening condition interactions were observed. Lack of a main effect of noise in adult listeners as well as a lack of an age effect on P300 amplitude is consistent with previous findings (Cranford and Martin, 1991; Salisbury et al, 2002). These findings suggest that the amount of neural substrate synchronously responding did not significantly change in noise compared with quiet conditions and also did not significantly change because of age. Thus, as task complexity increased with increasing noise level, cortical neurons contributing to the P300 did not significantly change for either the YA or OA groups. Salisbury et al (2002) explained a lack of P300 amplitude difference in noise to reflect a counteraction 
between the increase in resource allocation necessary for increased task complexity with the reduction in information transfer that occurs in noise. Researchers have since confirmed the reduction in amplitude of exogenous auditory cortical responses in noise (N1 and/or P2) (Billings et al, 2009; McCullagh et al, 2012; McCullagh and Shinn, 2013; Billings et al, 2015) suggesting the existence of the reduction in information transfer in noise that Salisbury et al (2002) discussed. Moreover, aging studies using fMRI and positron emission tomography to assess cognitive tasks have demonstrated increased bilateral activation in the brain (Reuter-Lorenz et al, 1999; Cabeza, 2001; Charroud et al, 2015) which may be related to the increase in resource allocation. Researchers have also suggested that frontal areas, specifically the prefrontal cortex, have increased activation in cognitive processing tasks and may be a compensatory mechanism in OAs (Fabiani and Friedman, 1995; Fabiani et al, 1998; Reuter-Lorenz and Cappell, 2008; van Dinteren et al, 2014). We hypothesize that perhaps compensatory mechanisms may have been activated in background noise in the OAs, thus reducing the influence of background noise on the P300 amplitude. Although not elicited in background noise, other researchers have not found significant age-related P300 amplitude differences with tonal target stimuli (Katayama and Polich, 1996; Gaál et al, 2007). Gaál et al (2007) did not demonstrate a significant interaction between age and task complexity on P300 amplitude which is similar to the current study. Katayama and Polich (1996) did not find significant P300 amplitude differences across differing P300 tasks (1-, 2-, and 3-tone oddball paradigms) which they explained was an indication that the P300 is an index of cognitive processing because changing the stimuli did not change the P300 amplitude.

It should also be noted that more variability in P300 amplitude existed for OAs as compared with YAs in the quiet condition that was not observed in the noise conditions. Fjell and Walhovd (2007) demonstrated trialto-trial variability in P300 amplitudes that positively correlated with age. It may be the case that there is an inherent variability in OAs that is difficult to control for when evaluating late auditory evoked potentials. In addition, sample size may be a contributory factor to the variability observed in the present investigation. A larger sample size may have reduced the variability and potentially yielded statistically significant differences in amplitude measurements. These results suggest that P300 amplitude is likely related to primarily endogenous, or cognitive, processing and that aging and background noise did not significantly impact the amplitude; however, exogenous contributions cannot be ruled out. Additional research is warranted to determine the relationships between endogenous and exogenous interaction, task complexity, resource allocation, and cognitive processing in YAs compared with OAs.

\section{CONCLUSION}

$\mathrm{T}$ his present investigation is unique in that agerelated changes are studied by eliciting the auditory P300 binaurally and at multiple SNRs. Results from the present study provide evidence that auditory cortical processing, regardless of age, is poorer at more difficult SNRs. However, it also demonstrates that OAs perform significantly poorer than their YA counterparts. This supports the notion that some degree of age-related decline in synchronous firing and rate of transmission of the auditory cortical neurons contributing to the auditory P300 exists. Further studies are needed to elucidate the impact of noise on auditory cortical processing across populations. Moreover, although the evaluation of the ability to process in noise is primarily focused on behavioral measures, objective measures such as those described in this protocol need to be created to provide additional means by which to evaluate processing in noise in clinical populations.

\section{REFERENCES}

Anderer P, Semlitsch HV, Saletu B. (1996) Multichannel auditory event-related brain potentials: effects of normal aging on the scalp distribution of N1, P2, N2 and P300 latencies and amplitudes. Electroencephalogr Clin Neurophysiol 99(5):458-472.

Anderson S, White-Schwoch T, Parbery-Clark A, Kraus N. (2013) A dynamic auditory-cognitive system supports speech-in-noise perception in older adults. Hear Res 300:18-32.

Bertoli S, Smurzynski J, Probst R. (2005) Effects of age, agerelated hearing loss, and contralateral cafeteria noise on the discrimination of small frequency changes: psychoacoustic and electrophysiological measures. J Assoc Res Otolaryngol 6(3): 207-222.

Billings CJ, Penman TM, McMillan GP, Ellis EM. (2015) Electrophysiology and perception of speech in noise in older listeners: effects of hearing impairment and age. Ear Hear 36(6):710-722.

Billings CJ, Tremblay KL, Stecker GC, Tolin WM. (2009) Human evoked cortical activity to signal-to-noise ratio and absolute signal level. Hear Res 254(1-2):15-24.

Brody H, Harman D, Ordy JM. (1975) Aging. In: Clinical, Morphologic, and Neuro-Chemical Aspects in the Aging Central Nervous System, Vol. 1. New York: Raven Press.

Cabeza R. (2001) Cognitive neuroscience of aging: contributions of functional neuroimaging. Scand J Psychol 42(3):277-286.

Carhart R, Jerger J. (1959) Preferred method for clinical determination of pure-tone thresholds. J Speech Hear Disord 16:340-345.

Charroud C, Steffener J, Le Bars E, Deverdun J, Bonafe A, Abdennour M, Portet F, Molino F, Stern Y, Ritchie K, Menjot de Champfleur N, Akbaraly TN. (2015) Working memory activation of neural networks in the elderly as a function of information processing phase and task complexity. Neurobiol Learn Mem 125: $211-223$.

Cranford JL, Martin DR. (1991) Age-related changes in binaural processing: I. Evoked potential findings. Am J Otol 12(5): 357-364. 
Dubno JR, Dirks DD, Morgan DE. (1984) Effects of age and mild hearing loss on speech recognition in noise. J Acoust Soc Am 76(1):87-96.

Elberling C, Bak C, Kofoed B, Lebech J, Saermark K. (1982) Auditory magnetic fields from the human cerebral cortex: location and strength of an equivalent current dipole. Acta Neurol Scand 65(6):553-569.

Fabiani M, Friedman D. (1995) Changes in brain activity patterns in aging: the novelty oddball. Psychophysiology 32(6):579-594.

Fabiani M, Friedman D, Cheng JC. (1998) Individual differences in P3 scalp distribution in older adults, and their relationship to frontal lobe function. Psychophysiology 35(6):698-708.

Fjell AM, Walhovd KB. (2007) Stability of brain potentials, mental abilities, and cortical thickness. Neuroreport 18(8):725-728.

Gaál ZA, Csuhaj R, Molnár M. (2007) Age-dependent changes of auditory evoked potentials-effect of task difficulty. Biol Psychol 76(3):196-208.

Goodin DS, Squires KC, Henderson BH, Starr A. (1978) Age-related variations in evoked potentials to auditory stimuli in normal human subjects. Electroencephalogr Clin Neurophysiol 44(4):447-458.

Goodin DS, Starr A, Chippendale T, Squires KC. (1983) Sequential changes in the P3 component of the auditory evoked potential in confusional states and dementing illnesses. Neurology 33(9): $1215-1218$.

Gordon-Salant S. (1987) Age-related differences in speech recognition performance as a function of test format and paradigm. Ear Hear 8(5):277-282.

Gordon-Salant S, Fitzgibbons PJ. (1997) Selected cognitive factors and speech recognition performance among young and elderly listeners. J Speech Lang Hear Res 40(2):423-431.

Greenhouse W, Geisser S. (1959) On methods in the analysis of profile data. Psychometrika 24:95-112.

Gregoire J, Van der Linden M. (1997) Effect of age on forward and backward digit spans. Aging Neuropsychol Cogn 4(2):140-149.

Helfer KS, Freyman RL. (2014) Stimulus and listener factors affecting age-related changes in competing speech perception. $J$ Acoust Soc Am 136(2):748-759.

Jirsa RE. (1992) The clinical utility of the P3 AERP in children with auditory processing disorders. J Speech Hear Res 35(4):903-912.

Katayama J, Polich J. (1996) P300 from one-, two-, and three-stimulus auditory paradigms. Int $J$ Psychophysiol 23(1-2):33-40.

Kibbe-Michal K, Verkest SB, Gollegly KM, Musiek FE. (1986) Late auditory evoked potentials and the P300. Hear Instr 37:22-24.

Kim JR, Ahn SY, Jeong SW, Kim LS, Park JS, Chung SH, Oh MK. (2012) Cortical auditory evoked potential in aging: effects of stimulus intensity and noise. Otol Neurotol 33(7):1105-1112.

Kropotov J, Ponomarev V, Tereshchenko EP, Müller A, Jäncke L. (2016) Effect of aging on ERP components of cognitive control. Front Aging Neurosci 8:69.

McCullagh J, Musiek FE, Shinn JB. (2012) Auditory cortical processing in noise in normal-hearing young adults. Audiol Med 10: $114-121$.

McCullagh J, Shinn JB. (2013) Auditory cortical processing in noise in younger and older adults. Hear Balance Commun 11: $182-190$
McCullagh J, Weihing J, Musiek F. (2009) Comparisons of P300s from standard oddball and omitted paradigms: implications to exogenous/endogenous contributions. J Am Acad Audiol 20(3): 187-195. quiz 219.

Obert AD, Cranford JL. (1990) Effects of neocortical lesions on the P300 component of the auditory evoked response. Am J Otol 11(6): $447-453$.

Papanicolaou AC, Loring DW, Eisenberg HM. (1984) Age-related differences in recovery cycle of auditory evoked potentials. Neurobiol Aging 5(4):291-295.

Peters A. (2002) The effects of normal aging on myelin and nerve fibers: a review. J Neurocytol 31(8-9):581-593.

Pichora-Fuller MK. (2003) Cognitive aging and auditory information processing. Int J Audiol 42:2S26-2S32.

Pichora-Fuller MK, Schneider BA, Daneman M. (1995) How young and old adults listen to and remember speech in noise. $J$ Acoust Soc Am 97(1):593-608.

Picton TW, Stuss DT, Champagne SC, Nelson RF. (1984) The effects of age on human event-related potentials. Psychophysiology 21(3):312-325.

Pfefferbaum A, Ford JM, Roth WT, Kopell BS. (1980) Age-related changes in auditory event-related potentials. Electroencephalogr Clin Neurophysiol 49(3-4):266-276.

Pfefferbaum A, Ford JM, Wenegrat BG, Roth WT, Kopell BS. (1984) Clinical application of the P3 component of event-related potentials. I. Normal aging. Electroencephalogr Clin Neurophysiol 59(2):85-103.

Polich J. (1987) Task difficulty, probability, and inter-stimulus interval as determinants of P300 from auditory stimuli. Electroencephalogr Clin Neurophysiol 68(4):311-320.

Polich J, Howard L, Starr A. (1985) Stimulus frequency and masking as determinants of P300 latency in event-related potentials from auditory stimuli. Biol Psychol 21(4):309-318.

Reuter-Lorenz PA, Cappell KA. (2008) Neurocognitive aging and the compensation hypothesis. Curr Dir Psychol Sci 17:177-182.

Reuter-Lorenz PA, Stanczak L, Miller AC. (1999) Neural recruitment and cognitive aging: two hemispheres are better than one, especially as you age. Psychol Sci 10(6):494-500.

Rossini PM, Rossi S, Babiloni C, Polich J. (2007) Clinical neurophysiology of aging brain: from normal aging to neurodegeneration. Prog Neurobiol 83(6):375-400.

Salisbury DF, Desantis MA, Shenton ME, McCarley RW. (2002) The effect of background noise on P300 to suprathreshold stimuli. Psychophysiology 39(1):111-115.

Scherg M, Vajsar J, Picton TW. (1989) A source analysis of the late human auditory evoked potentials. J Cogn Neurosci 1(4): $336-355$.

Scherg M, Von Cramon D. (1986) Evoked dipole source potentials of the human auditory cortex. Electroencephalogr Clin Neurophysiol 65(5):344-360.

Snell KB, Mapes FM, Hickman ED, Frisina DR. (2002) Word recognition in competing babble and the effects of age, temporal processing, and absolute sensitivity. J Acoust Soc Am 112(2):720-727.

Squires KC, Hecox KE. (1983) Electro-physiological evaluation of higher level auditory processing. Semin Hear 4:415-432. 
Stapleton JM, Halgren E. (1987) Endogenous potentials evoked in simple cognitive tasks: depth components and task correlates. Electroencephalogr Clin Neurophysiol 67(1):44-52.

Stapleton JM, O’Reilly T, Halgren E. (1987) Endogenous potentials evoked in simple cognitive tasks: scalp topography. Int $J \mathrm{Neu}$ rosci 36(1-2):75-87.

Stuart A, Phillips DP. (1996) Word recognition in continuous and interrupted broadband noise by young normal-hearing, older normalhearing, and presbyacusic listeners. Ear Hear 17(6):478-489.

Sutton S, Braren M, Zubin J, John ER. (1965) Evoked-potential correlates of stimulus uncertainty. Science 150(3700):1187-1188.
Tun PA, O'Kane G, Wingfield A. (2002) Distraction by competing speech in young and older adult listeners. Psychol Aging 17(3): $453-467$.

van Dinteren R, Arns M, Jongsma MLA, Kessels RP. (2014) Combined frontal and parietal P300 amplitudes indicate compensated cognitive processing across the lifespan. Front Aging Neurosci 6:294.

Vaughan HG Jr, Ritter W. (1970) The sources of auditory evoked responses recorded from the human scalp. Electroencephalogr Clin Neurophysiol 28(4):360-367.

Weihing J, Musiek FE. (2008) An electrophysiological measure of binaural hearing in noise. $J$ Am Acad Audiol 19(6):481-495. 